\title{
Neurons in red nucleus and primary motor cortex exhibit similar responses to mechanical perturbations applied to the upper-limb during posture
}

\author{
Troy M. Herter ${ }^{1,2}$, Tomohiko Takei ${ }^{1}$, Douglas P. Munoz ${ }^{1,3}$ and Stephen H. Scott ${ }^{1,3,4 *}$ \\ ${ }^{1}$ Centre for Neuroscience Studies, Queen's University, Kingston, ON, Canada, ${ }^{2}$ Department of Exercise Science, University \\ of South Carolina, Columbia, SC, USA, ${ }^{3}$ Department of Biomedical and Molecular Sciences, Queen's University, Kingston, \\ ON, Canada, ${ }^{4}$ Department of Medicine, Queen's University, Kingston, ON, Canada
}

\section{OPEN ACCESS}

Edited by:

Elizabeth B. Torres,

Rutgers University, USA

Reviewed by:

Sergiy Yakovenko,

West Virginia University, USA

Paul David Cheney,

University of Kansas Medical Center,

USA

${ }^{*}$ Correspondence:

Stephen H. Scott,

Centre for Neuroscience Studies,

Queen's University, Botterell Hall,

Room 219, Kingston, ON K7P 3N6,

Canada

Tel: (613) 533-2855,

Fax: (613) 533-6840

steve.scott@queensu.ca

Received: 14 October 2014 Accepted: 29 March 2015

Published: 24 April 2015

Citation:

Herter TM, Takei T, Munoz DP and

Scott SH (2015) Neurons in red

nucleus and primary motor cortex

exhibit similar responses to

mechanical perturbations applied to

the upper-limb during posture.

Front. Integr. Neurosci. 9:29.

doi: 10.3389/fnint.2015.00029
Primary motor cortex (M1) and red nucleus (RN) are brain regions involved in limb motor control. Both structures are highly interconnected with the cerebellum and project directly to the spinal cord, although the contribution of $\mathrm{RN}$ is smaller than M1. It remains uncertain whether $\mathrm{RN}$ and $\mathrm{M} 1$ serve similar or distinct roles during posture and movement. Many neurons in M1 respond rapidly to mechanical disturbances of the limb, but it remains unclear whether RN neurons also respond to such limb perturbations. We have compared discharges of single neurons in RN $(n=49)$ and M1 $(n=109)$ of one monkey during a postural perturbation task. Neural responses to whole-limb perturbations were examined by transiently applying $(300 \mathrm{~ms})$ flexor or extensor torques to the shoulder and/or elbow while the monkeys attempted to maintain a static hand posture. Relative to baseline discharges before perturbation onset, perturbations evoked rapid ( $<100 \mathrm{~ms}$ ) changes of neural discharges in many $\mathrm{RN}$ (28 of 49,57\%) and M1 (43 of $109,39 \%)$ neurons. In addition to exhibiting a greater proportion of perturbationrelated neurons, RN neurons also tended to exhibit higher peak discharge frequencies in response to perturbations than M1 neurons. Importantly, neurons in both structures exhibited similar response latencies and tuning properties (preferred torque directions and tuning widths) in joint-torque space. Proximal arm muscles also displayed similar tuning properties in joint-torque space. These results suggest that $\mathrm{RN}$ is more sensitive than $\mathrm{M} 1$ to mechanical perturbations applied during postural control but both structures may play a similar role in feedback control of posture.

Keywords: red nucleus, primary motor cortex, upper-limb muscle, perturbation, optimal feedback control

\section{Introduction}

It is well established that primary motor cortex (M1) and red nucleus (RN) form parallel pathways for motor control as both structures have axonal projections to the spinal cord including direct connections to motoneurons (Fetz and Cheney, 1980; Buys et al., 1986; Cheney et al., 1991; Mewes and Cheney, 1991, 1994; Belhaj-Saif et al., 1998; McKiernan et al., 1998; Park et al., 2004). Furthermore, M1 and RN, together with the cerebellum, form an extensively 
interconnected premotor network involved in the control of upper limb movement (reviewed in Kennedy, 1990; Houk et al., 1993; Keifer and Houk, 1994). Understanding the common and distinct contributions of $\mathrm{M} 1$ and $\mathrm{RN}$ is important to our understanding of volitional motor control.

The patterns of activity observed in RN are generally similar to those observed in M1. Neural activity M1 is correlated with the timing and magnitude of upper-limb muscle activity (Smith et al., 1975; Bennett and Lemon, 1996; Scott, 1997; Holdefer and Miller, 2002), as is the activity of neurons in RN (Miller et al., 1993; Mewes and Cheney, 1994; Miller and Houk, 1995; Belhaj-Saif et al., 1998; Miller and Sinkjaer, 1998). Neural activity in M1 can reflect either kinematic (motion) or kinetic (forces) features of movement (reviewed in Scott, 2003), a feature that is also observed in RN (Kohlerman et al., 1982; Gibson et al., 1985a,b; Kennedy, 1987; Cheney et al., 1988; Mewes and Cheney, 1994). More recent studies suggest that RN may be specialized for controlling grasping movements coupled with reaching (Sinkjaer et al., 1995; van Kan and McCurdy, 2001, 2002a,b).

A recent hypothesis proposes that the volitional motor system may act like an optimal feedback controller (Todorov and Jordan, 2002; Todorov, 2004). This framework highlights the importance of afferent feedback for voluntary control of movement and predicts that feedback will be modified based on the goal of the behavioral task (Scott, 2004, 2012). Examination of muscle stretch responses highlight that the long-latency response is modified by limb mechanics (Kurtzer et al., 2008, 2009, 2014), motor intention (Pruszynski et al., 2008; Dimitriou et al., 2012; Crevecoeur et al., 2013), motor learning (Cluff and Scott, 2013), and features of the goal and environment (Nashed et al., 2012, 2014; Omrani et al., 2013). The fact that these context dependent responses occur during long, but not short latency responses is significant because it suggests that they are generated supraspinally.

Supraspinal involvement in feedback control of volitional movement is also supported by electrophysiological studies of M1 neurons in awake, behaving monkeys. These studies have observed that M1 neurons respond to passive joint motion (Fetz et al., 1980; Lemon, 1981; Scott, 1997; Scott and Kalaska, 1997) and exhibit rapid responses to mechanical perturbations applied to a single (Evarts, 1973; Evarts and Fromm, 1977; Wolpaw, 1980a; Flament and Hore, 1988; Bauswein et al., 1991) or multiple joints (Herter et al., 2009). Importantly, perturbation responses in M1 consider the influence of limb mechanics (Pruszynski et al., 2011b), motor intention (Conrad et al., 1974, 1975; Evarts and Tanji, 1974; Wolpaw, 1980b; Pruszynski et al., 2014), and whether the animal is actively engaged in a motor task (Omrani et al., 2014). Furthermore, rapid responses to perturbations have been observed in M1 neurons with identified projections to the pyramidal tract (Evarts and Tanji, 1976; Fromm et al., 1984), including M1 neurons with direct connections onto spinal motor neurons (Cheney and Fetz, 1984).

It remains unclear, however, whether neurons in monkey $\mathrm{RN}$ exhibit rapid motor responses similar to those observed in M1. Some studies have found that most neurons in $\mathrm{RN}$ respond to passive joint movements (Larsen and Yumiya, 1980) and torque perturbations (Mewes and Cheney, 1994) of the upper-limb. However, other studies have found that sensory stimulation evokes weak or negligible responses in RN neurons (Gibson et al., 1985a; Kennedy et al., 1986). The present study uses a multijoint paradigm to investigate whether mechanical perturbations evoke rapid sensorimotor responses in $\mathrm{RN}$ neurons that are similar to those observed in M1 and upper-limb muscles. We hypothesized that neurons in $\mathrm{RN}$ would exhibit rapid responses to mechanical perturbations with directional tuning features that are similar to M1 neurons and upper-limb muscles. To test this hypothesis, we compared rapid responses of $\mathrm{RN}$ neurons, M1 neurons and upper-limb muscles evoked by multi-joint perturbations (transient mechanical torques at elbow and/or shoulder joints) applied while monkeys maintained a constant arm posture. Here we show that directional tuning features of $\mathrm{RN}$ and M1 neurons were similar to those observed in upperlimb muscles.

\section{Methods}

\section{Subjects and Apparatus}

Four male rhesus monkeys (Macaca mulatta, 6-10 kg) were trained to perform whole-limb visuomotor tasks while wearing KINARM (BKIN Technologies, Kingston, ON, Canada), a robotic exoskeleton that supports the arm, permits planar shoulder and elbow motion, and can apply mechanical torques at the shoulder and/or elbow (Scott, 1999; Figure 1A). A virtual reality system presented visual targets within the limb's movement plane while permitting the monkeys to view their entire limb. The Queen's University Animal Care Committee approved all procedures.

\section{Behavioral Task}

The monkeys performed a postural perturbation task (Herter et al., 2009). Mechanical perturbations were transiently applied to the monkeys' right arms while they maintained their right hand at a visual target (6 $\mathrm{mm}$ radius) displayed near the center of the arm's workspace (30 $0^{\circ}$ shoulder flexion, $90^{\circ}$ elbow flexion) where passive viscoelastic forces are relatively small (Graham et al., 2003). The monkeys initiated each trial by moving their right hand to the visual target and holding it within an acceptance window (8 mm radius) for 1000-1500 ms. One of nine perturbations was then transiently applied to the monkeys' arm for $300 \mathrm{~ms}$. The nine perturbation conditions included four single-joint torques (shoulder flexion, SF; shoulder extension, $S E$; elbow flexion, $E F$; elbow extension, $E E$ ), four multi-joint torques $(S F+E F, S F+E E, S E+E F, S E+E E)$, and an unloaded condition (Figure 1B). The magnitude of torque applied at each joint was fixed at either $\pm 0.12 \mathrm{Nm}$ (Monkeys A-C) or $\pm 0.32 \mathrm{Nm}$ (Monkey D), producing a uniform distribution in joint-torque space but a torque magnitude that was $\sqrt{ } 2$ greater in multi-joint than single-joint conditions. Each perturbation (except the unloaded condition) pushed the monkeys' hand from the target's acceptance window and the monkeys were required to return their hand to the visual target within $1500 \mathrm{~ms}$ and hold it there for 
A

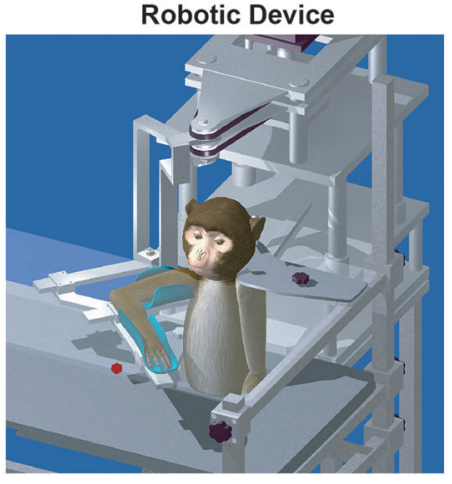

B

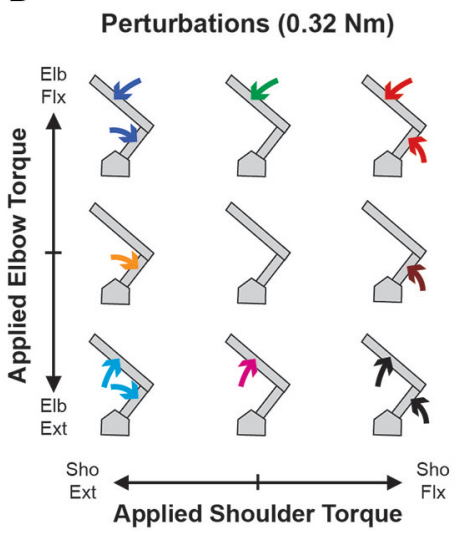

C

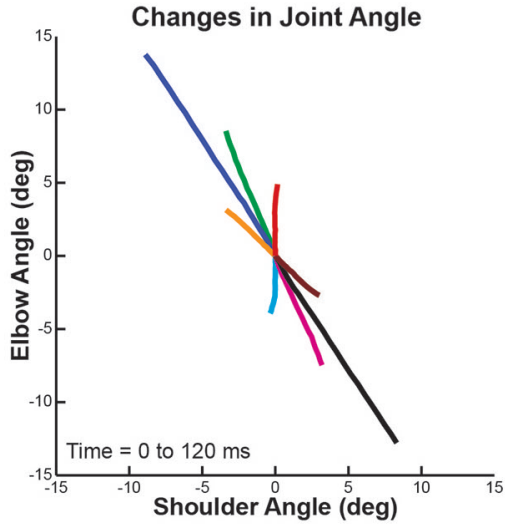

FIGURE 1 | Robotic device, perturbation conditions and perturbation-evoked kinematics. (A) Schematic representation of the KINARM exoskeleton robot used in the study. (B) Arrangement of the nine perturbation conditions applied to the monkey's upper-limb. Joint torques imposed at the shoulder and elbow joints are represented along the $x$ and $y$ axes, respectively (joint-torque space: flexor torque positive and extensor torque negative). Modified from Herter et al. (2009). (C) Joint motion evoked by each perturbation condition. Changes in shoulder and elbow angle in first 120 ms after perturbation onset are represented along the $x$ and $y$ axes, respectively (flexion positive and extension negative). Colors of each line are associated with the respective perturbation conditions in (B). Modified from Herter et al. (2009). another 1000-1500 ms to receive a liquid reward. The nine perturbation conditions were presented in a pseudo-random block design with each block repeated five times for a total of 45 trials.

\section{Data Collection}

Neural data was collected from the left RN of one monkey (Monkey D) using standard extracellular recording techniques developed for recording from brainstem structures (Marino et al., 2008). Microelectrodes were advanced through guide tubes that were placed inside a grid mounted within a stainless steel recording chamber (Crist Instruments, Hagerstown, MD) that was implanted over the stereotaxic coordinates of the RN. The recording chamber was centered on the midline and angled $35^{\circ}$ posterior of vertical, which allowed us to identify the superior and inferior colliculus during penetrations into the rostral and caudal $\mathrm{RN}$, respectively. Neural data was collected from the left M1 of all four monkeys using standard extracellular recording techniques for cortical neurons (Herter et al., 2007). However, M1 data from Monkey D only is presented in the current report because RN data was collected from Monkey D only. For penetrations into both $\mathrm{RN}$ and $\mathrm{M} 1$, microelectrodes were advanced until neural activity was observed in response to active or passive arm movements. Single neurons were then isolated and neural activity was recorded from all neurons that were related to active or passive movements of the shoulder and/or elbow, but not the wrist and/or fingers.

Electromyographic (EMG) activity was collected from proximal arm muscles involved in flexion or extension at the shoulder and/or elbow (Graham and Scott, 2003) using standard techniques (Loeb and Gans, 1986; Kurtzer et al., 2006a). Acute recordings were obtained from all four monkeys using pairs of single-strand wires that were percutaneously inserted approximately $5 \mathrm{~mm}$ apart in the muscle belly. Chronic recordings were attained from Monkeys A and C using bipolar multi-strand electrodes that were subcutaneously implanted within the superficial muscle belly. EMG activity was recorded from 11 different upper-limb muscles, including shoulder flexors (Anterior Deltoid, Pectoralis Major), shoulder extensors (Posterior Deltoid, Medial Deltoid), elbow flexors (Brachialis, Brachioradialis, Extensor Carpi Radialis Longus), elbow extensors (Triceps lateral head, Triceps medial head), biarticular flexors (Biceps long head, Biceps short head), and biarticular extensors (Triceps long head, Dorsoepitrochlearis). Electrode placement in each muscle was verified using micro-stimulation through the recording electrode.

During recording sessions, EMG signals were band-pass filtered (100-3,000 Hz) and recorded at $1 \mathrm{kHz}$ (Monkeys A-C) or $4 \mathrm{kHz}$ (Monkey D). During the subsequent offline analysis, signals were full-wave rectified and integrated into $5 \mathrm{~ms}$ bins. Muscles were only included in the analyses if they obtained a score of $\geq 3$ on a subjective rating scale of signal quality ( $1=$ poor, 5 = excellent; Kurtzer et al., 2006a). EMG data from the four monkeys was included in our analyses for the current report.

Joint angles, velocities, and applied torques were recorded at $1 \mathrm{kHz}$ (Monkeys A-C) or $4 \mathrm{kHz}$ (Monkey D). Cartesian hand positions and tangential hand speed were calculated from joint angles and velocities.

\section{Data Analyses Neural Activity}

Analyses of RN and M1 neurons were restricted to perturbationrelated neurons, defined as neurons that: (1) exhibited onset latencies between 10 and $100 \mathrm{~ms}$ after perturbation onset; and (2) exhibited significant directional-tuning in joint-torque space during the epoch lasting from 20 to $120 \mathrm{~ms}$ after perturbation onset. Onset latencies were obtained from spike frequencies that were averaged across the three spatially adjacent perturbation 
conditions with the highest mean activity during the postperturbation epoch ( $n=15$ trials). Spike frequencies that were calculated at $5 \mathrm{~ms}$ intervals with an asymmetric spike density filter (Thompson et al., 1996; Herter et al., 2009). Each neural spike was convolved with a double exponential kernel that mimics a post-synaptic potential ( $1 \mathrm{~ms}$ rise and $20 \mathrm{~ms}$ fall). Onset latency was determined as the first time that spike frequency increased for at least three consecutive points $(15 \mathrm{~ms})$ and extended beyond $4 \mathrm{SD}$ of the mean during the period of $100 \mathrm{~ms}$ preceding perturbation onset.

Directional tuning in joint-torque space ( $\mathrm{SF}=0^{\circ}, \mathrm{EF}=90^{\circ}$, $\mathrm{SE}=180^{\circ}, \mathrm{EE}=270^{\circ}$ ) was obtained by examining changes in neural activity as a function of perturbation direction in joint-torque space. Directional tuning features were calculated with the plate method, which describes several features of directional tuning without assuming an underlying tuning function (Gribble and Scott, 2002). This method characterizes the "mass distribution" of torque-related activity by assuming that activity changes linearly between sampled torque directions and that torque magnitude is equal for each torque direction. To use this method, the lowest activity across all trials was subtracted so that all values were greater or equal to zero. Significance of directional tuning was determined using a nonparametric "bootstrapping" test (Scott and Kalaska, 1997), in which the distance of the center mass from the origin (i.e., magnitude of the centroid) was compared with bootstrap values of the centroid obtained by randomly reassigning the neural activity across all trials. A neuron was considered to have significant directional tuning if fewer than 100 of 10,000 bootstrap values of the centroid were greater than the actual value of the centroid $(p<0.01)$.

For all perturbation-related neurons, the centroid was used to calculate four directional tuning features (Herter et al., 2007). (1) Preferred-torque direction (PTD), which describes the angle associated with the greatest increase in activity, was calculated as the direction of the centroid relative to the origin in joint-torque space. (2) Torque-slope (TS), which expresses the sensitivity to loads, was calculated by normalizing the magnitude of the centroid by the torque magnitude $(0.32 \mathrm{Nm})$. (3) Tuning Width was calculated as the ratio of changes in activity perpendicular to the PTD axes relative to changes in activity along the PTD axes. This method of computing tuning width yields values ranging from 0 (narrow) to 1 (broad), where a cosine obtains a tuning width of 0.44. (4) Excitation-Inhibition Ratio (EIR) describes the relationship between changes in activity (relative to the unloaded baseline condition) for the load condition nearest to the preferred-torque direction $(\triangle \mathrm{PTD})$ and for the load condition opposite the preferred-torque direction $(\triangle \mathrm{OPP})$. EIRs were computed as:

$$
E I R=\frac{(\Delta \mathrm{PTD}+\Delta \mathrm{OPP})}{(\Delta \mathrm{PTD}-\Delta \mathrm{OPP})}
$$

Note that changes in activity relative to the unloaded baseline condition are generally excitatory (positive) for load conditions near the PTD and inhibitory (negative) for load conditions opposite the PTD. As a result, EIRs values typically range from -1 to 1 , where positive (negative) EIRs occur when the magnitude of excitation at the PTD is greater (lesser) than the magnitude of inhibition opposite the preferredtorque direction. Values near 0 occur when the magnitudes of excitation and inhibition are similar. In some cases, EIRs can go beyond $1(-1)$ if both $\triangle \mathrm{PTD}$ and $\triangle \mathrm{OPP}$ exhibit excitation or inhibition.

Rayleigh tests were used to determine if distributions of PTDs were statistically unimodal or bimodal relative to a uniform distribution (Batschelet, 1981). This statistic is based on mean vector length, which describes similarity across a sample of angles (e.g., PTDs). A mean vector length of 0 is obtained if all angles are uniformly distributed and a value of 1 is obtained if all angles are identical. The value of a mean vector length along this continuum provides an index that is compared with a Rayleigh distribution. For a population with a significantly unimodal distribution, the mean orientation of the distribution determines the preferred direction of the population. For the bimodal Rayleigh test, all PTDs are multiplied by two, which creates a unimodal distribution if the underlying distribution is symmetrically bimodal. For a population with a significantly bimodal distribution, a preferred axis is obtained by dividing the average orientation by two.

\section{Muscle Activity}

To compare and contrast the patterns of activity of RN and M1 neurons with proximal arm muscles, the preceding analyses were also carried out on the EMG activity of our sample of proximal arm muscles. Note that TSs of muscles could not be directly compared with TSs obtained from neurons because muscle EMG was an arbitrary unit.

\section{Statistical Comparisons}

Onset latencies and PTDs of RN and M1 neurons were compared statistically with those of upper-limb muscles using $t$-tests $(p<0.05)$. For PTDs with a significant bimodal distribution, we multiplied each PTD by two to produce unimodal distributions that could be quantitatively compared with $t$-tests.

Assuming that activation of neurons in $\mathrm{RN}$ and M1 initiate muscle activity that produces movement, we expected the onset latencies of RN and M1 neurons would be shorter than onset latencies of upper limb muscles but similar to each other. Given similarities in their anatomical connections with the motor periphery (see Discussion), we also predicted that RN neurons, M1 neurons, and upper-limb muscles would exhibit similar bimodal distributions of PTDs biased towards whole limb flexor torques (elbow flexor and shoulder extensor) and whole limb extensor torques (elbow extensor and shoulder flexor). Given our a priori predictions, we did not correct for multiple comparisons for these tests of onset latencies and PTDs.

To capture the temporal evolution of neural and muscular activities, means were computed for each of the directional tuning properties (TSs, tuning widths, EIRs) in five $20 \mathrm{~ms}$ bins between 20 and $120 \mathrm{~ms}$. Each of these tuning features was then compared statistically using two-way $(3 \times 5)$ ANOVA $(p<0.05)$ that examined the effects of cell population (RN, M1, muscle) and temporal epoch (20-40 ms, $40-60 \mathrm{~ms}, 60-80 \mathrm{~ms}, 80-100 \mathrm{~ms}$, $100-120 \mathrm{~ms})$. We did not have any strong a priori predictions regarding these directional-tuning properties, thus we used the 
Bonferroni method (alpha divided by the number of $t$-tests) to correct for multiple comparisons.

Prior to statistical testing, onset latencies and directional tuning properties (PTDs, TSs, tuning widths, EIRs) of RN and M1 neurons and upper limb muscles were examined for normality using Lilliefors' test $(p<0.05)$. When necessary, parametric statistics (e.g., $t$-tests and ANOVAs) were replaced with equivalent nonparametric statistical tests (e.g., Wilcoxon rank sum tests and Freidman's tests).

\section{Results}

\section{Kinematics of the Perturbation Task}

Although the applied loads were uniformly distributed in jointtorque space (Figure 1B), joint motion was highly nonuniform due to intersegmental dynamics (Figure 1C). Over the first $120 \mathrm{~ms}$, each single-joint torque produced multi-joint motion (brown, green, orange and magenta lines) and two of the multijoint torques generated single-joint motion (red and cyan lines). In addition, the magnitude of joint motion resulting from these two multi-joint torques was much smaller than the other two multi-joint perturbation conditions (blue and black lines).

\section{Responses of RN Neurons to Perturbations}

We examined the activity of 49 neurons recorded in the upper-limb region of $\mathrm{RN}$ of Monkey D. After the recording sessions were completed, the monkey was euthanized and the brainstem was removed and sectioned for histological examination. Based on the location of the recording tracks, we confirmed that some penetrations targeted the RN. Over half of these neurons $(n=28,57 \%)$ exhibited perturbationrelated activity; i.e., their activity was significantly modulated at relatively short latencies (onset latency of 20-100 ms) and exhibited significant directional tuning in joint-torque space (bootstrap test, $p<0.01$ ). Figures $2 \mathrm{~A}, \mathbf{B}$, illustrate spike rasters and spike frequency histograms showing perturbation-related activity of two exemplar RN neurons. Both neurons showed markedly greater increases in activity for some perturbations conditions than others. The first neuron responded greatest to perturbations that required the monkey to generate an extensor torque at the shoulder and a flexor torque at the elbow (PTD $=139^{\circ}$, Figure $2 \mathrm{C}$ ). The second neuron was most sensitive when the monkey produced extensor torques at the elbow and flexor torques at the shoulder $\left(\mathrm{PTD}=290^{\circ}\right.$, Figure 2D). Both neurons displayed large differences in modulation between preferred and non-preferred perturbation conditions, which resulted in substantial TSs of 120 and 115 $(\mathrm{sp} / \mathrm{s}) / \mathrm{Nm}$, respectively (Figures 2C,D). Despite this similarity, the first neuron exhibited increases in activity for several perturbation conditions and decreases in a few directions, resulting in a tuning width that was slightly greater than cosine tuning (tuning width $=0.56$, Figure 2C, right). In
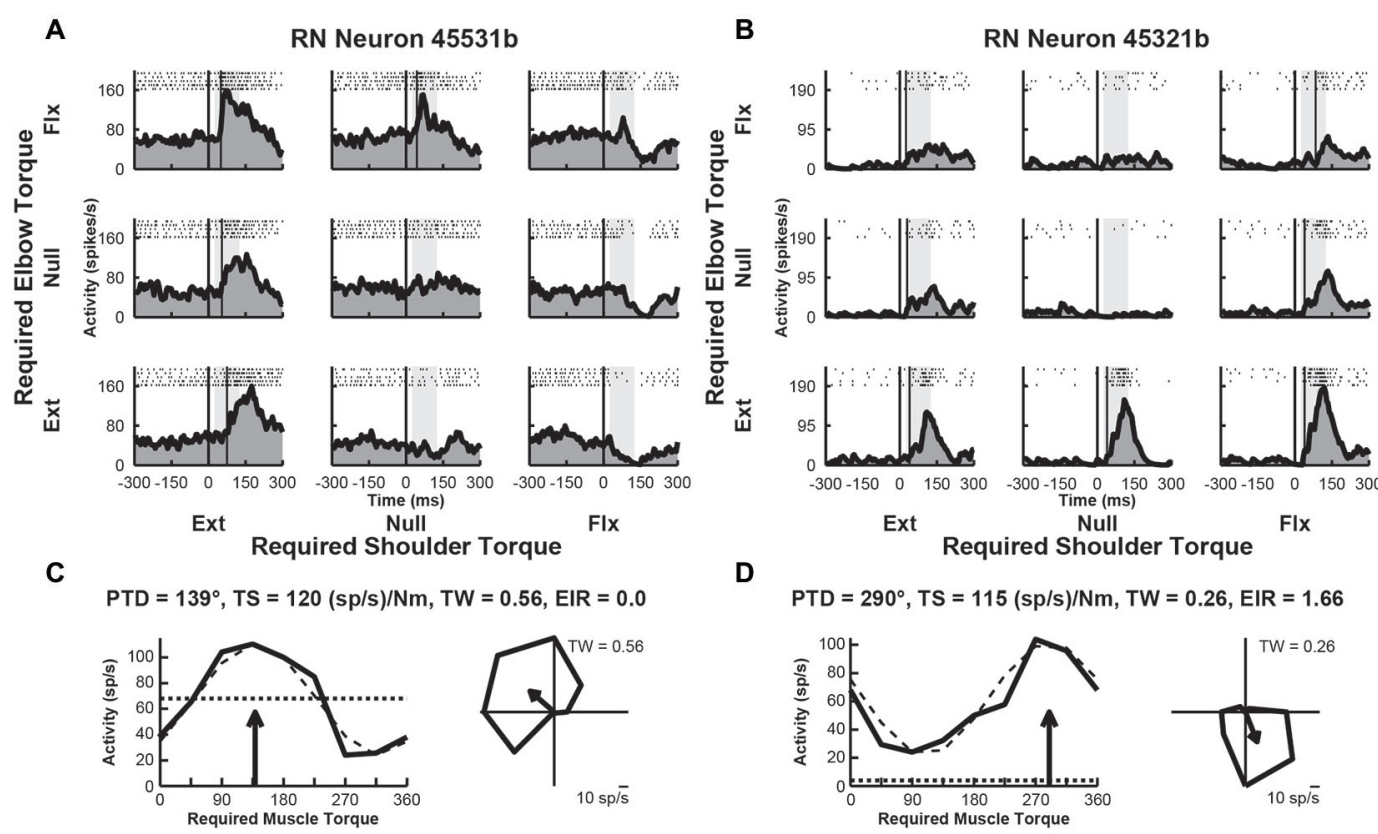

FIGURE 2 | Activity of exemplar RN neurons. (A) Rasters and histograms for each perturbation condition (arranged in joint-torque space) displaying the activity of an $\mathrm{RN}$ neuron that responded maximally to perturbations that required an extensor torque at the shoulder and a flexor torque at the elbow to counter the applied torques. (B) Activity of a $\mathrm{RN}$ neuron that responded maximally to perturbations that required a flexor torque at the shoulder and an extensor torque at the elbow. (C,D)
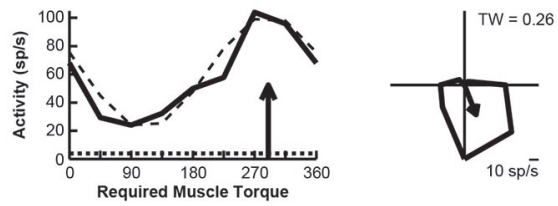

Directional tuning of the corresponding RN neurons. Left sub-panels illustrate linear plots of overall activity vs. joint-torque angle. Unloaded baseline activities and cosine fits are shown as dotted and dashed lines, respectively. Right sub-panels show polar plots of the perturbation-related activity (baseline removed) in joint-torque space. PTD, preferred-torque direction; TS, torque-slope; TW, tuning width; EIR, excitation-inhibition ratio. 
contrast, the second neuron showed large increases in activity for only a few perturbation conditions, resulting in narrow tuning relative to a cosine (tuning width $=0.26$, Figure $2 \mathrm{D}$, right). Relative to the unloaded baseline condition, the first neuron also showed similar increases (excitation) and deceases (inhibition) in activity in response to the various perturbations (excitation-inhibition ratio $=0.0$, Figure $2 \mathrm{C}$, left). The second neuron, however, exhibited excitation in response to each perturbation, though the extent of excitation differed between the perturbation directions (excitation-inhibition ratio $=1.66$, Figure 2D, left).

\section{Responses of M1 Neurons to Perturbations}

We examined the activity of 109 neurons recorded in the shoulder-elbow region of M1 of Monkey D. Data from these neurons were presented in a previous publication that compared the activity of M1 neurons in the current task with their activity during static postural maintenance (Herter et al., 2009). Close to half of these neurons $(n=43,39 \%)$ displayed perturbationrelated activity (onset latency, 20-100 ms; bootstrap test, $p<0.01$ ). Figures 3A,B, illustrate spike rasters and spike frequency histograms showing perturbation-related activity of two exemplar M1 neurons. Similar to the exemplar RN neurons seen previously, both exemplar M1 neurons showed large increases in activity for some perturbations. The first M1 neuron responded greatest to perturbations that required the monkey to generate an extensor torque at the shoulder and a flexor torque at the elbow (PTD $=114^{\circ}$, Figure $3 \mathrm{C}$ ). The second neuron was most sensitive for loads that required production of extensor torques at the elbow $\left(\mathrm{PTD}=283^{\circ}\right.$, Figure 3D). Compared to the exemplar $\mathrm{RN}$ neurons, the first M1 neuron displayed smaller differences in modulation between preferred and non-preferred perturbation conditions (torque-slope $=62(\mathrm{sp} / \mathrm{s}) / \mathrm{Nm}$, Figure $3 \mathrm{C})$. The second M1 neuron was more sensitive to loads though still less sensitive than the two RN neurons (torque-slope $=91(\mathrm{sp} / \mathrm{s}) / \mathrm{Nm}$, Figure 3D). Similar to the second RN neuron, both M1 neurons exhibited tuning widths that were slightly narrower than a cosine (tuning widths $=0.35$ and 0.28 , Figures 2C,D, right). Both M1 neurons showed similar diversity of excitation and inhibition that was seen in the exemplar RN neurons. Relative to baseline, the first M1 neuron showed increases and deceases in activity in response to the various perturbations, though excitation was greater than inhibition (excitation-inhibition ratio $=0.36$, Figure $3 \mathrm{C}$, left). Like the second $\mathrm{RN}$ neuron, the second M1 neuron exhibited excitation in response to each perturbation, though the extent of excitation varied across perturbation directions (excitation-inhibition ratio $=0.0$, Figure 3D, left).

\section{Responses of Upper-Limb Muscles to Perturbations}

We examined the activity of 33 EMG samples recorded from 33 different sites ( 1 sample per site) in 11 proximal arm muscles of

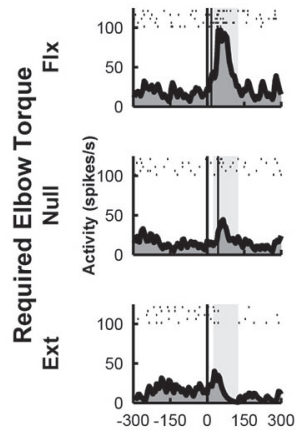

Ext

$\mathrm{PTD}=114^{\circ}, \mathrm{TS}=62(\mathrm{sp} / \mathrm{s}) / \mathrm{Nm}, \mathrm{TW}=0.35, \mathrm{EIR}=0.36$
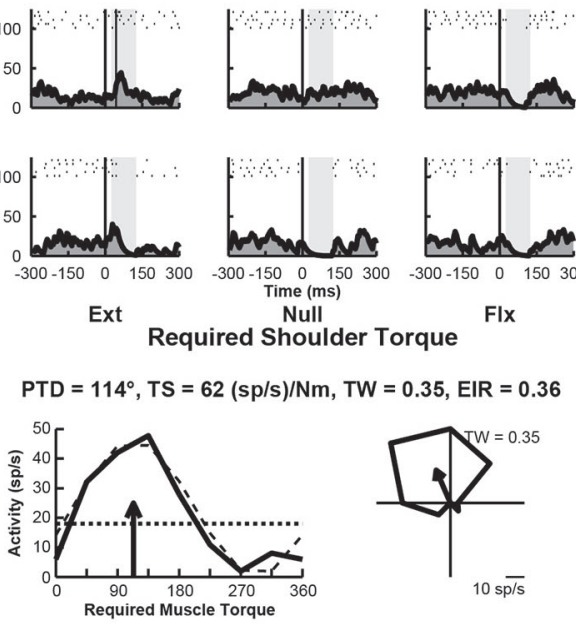

FIGURE 3 | Activity of exemplar M1 neurons. (A) Rasters and histograms for each perturbation condition (arranged in joint-torque space) displaying the activity of an M1 neuron that responded maximally to perturbations that required a flexor torque at the elbow. (B) Activity of a neuron that responded maximally to perturbations that required an extensor torque at the elbow. $(\mathbf{C}, \mathbf{D})$ Directional tuning of the
B
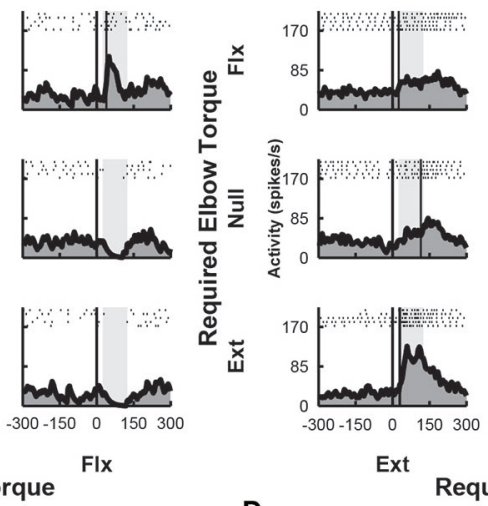

M1 Neuron $49032 c$
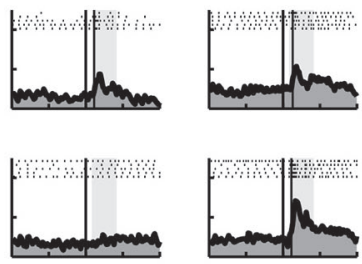

Ex

Required Shoulder Torque

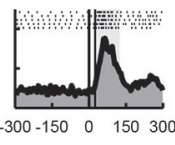

$\mathrm{FIx}$

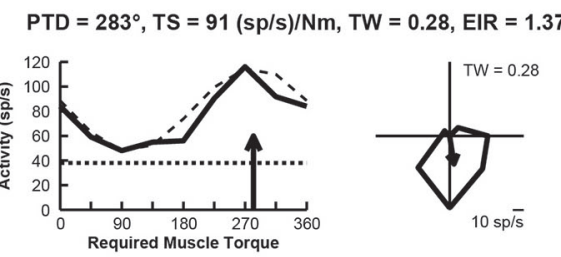

corresponding M1 neurons. Left sub-panels illustrate linear plots of overall activity vs. joint-torque angle. Unloaded baseline activities and cosine fits are shown as dotted and dashed lines, respectively. Right sub-panels show polar plots of the perturbation-related activity (baseline removed) in joint-torque space. PTD, preferred-torque direction; TS, torque-slope; TW, tuning width; EIR, excitation-inhibition ratio. 


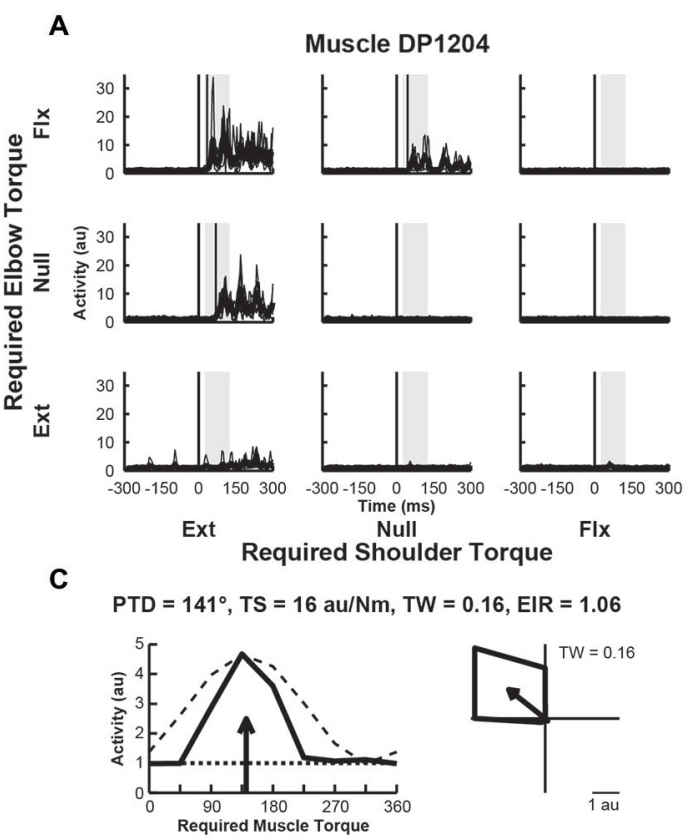

FIGURE 4 | Activity of exemplar upper-limb muscles.

(A) Electromyographic (EMG) activity of a posterior deltoid sample in each perturbation condition (arranged in joint-torque space). The posterior deltoid sample responded maximally to perturbations that required an extensor torque at the shoulder and a flexor torque at the elbow.

(B) EMG Activity of a brachioradialis sample that responded maximally to perturbations that required a flexor torque at the elbow. (C,D) Directional
B
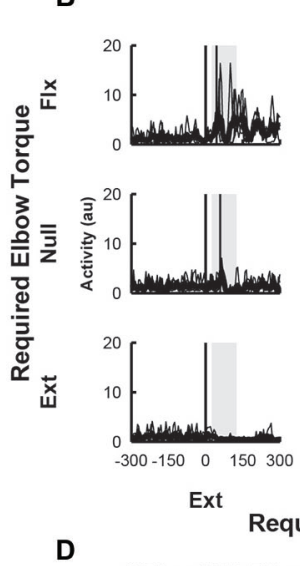

Muscle Br4308
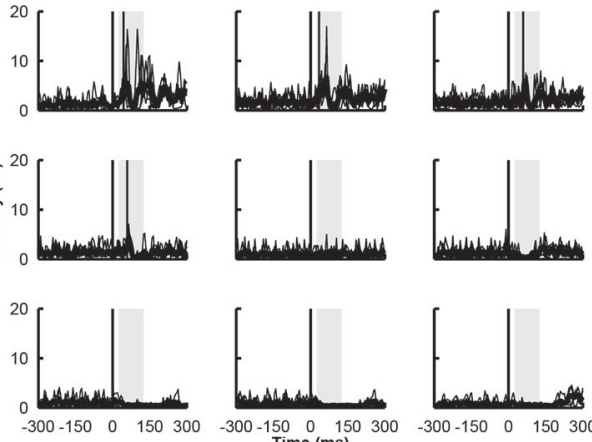

Ext

Required Shoulder Torque

FIx
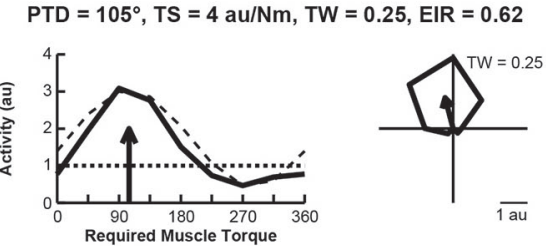

tuning of the corresponding muscle samples. Left sub-panels illustrate linear plots of overall activity vs. joint-torque angle. Unloaded baseline activities and cosine fits are shown as dotted and dashed lines, respectively. Right sub-panels show polar plots of the perturbation-related activity (baseline removed) in joint-torque space. PTD, preferred-torque direction; TS, torque-slope; TW, tuning width; EIR,

excitation-inhibition ratio.
Monkeys A-D. We found that two thirds of the muscles $(n=22$, $67 \%$ ) exhibited perturbation-related activity (onset latency, 20-100 ms; bootstrap test, $p<0.01$ ). Figures $4 \mathbf{A}, \mathbf{B}$, illustrate perturbation-related activity obtained from two exemplar upperlimb muscles. An EMG recording from a posterior deltoid sample showed increases in activity in response to loads that required production of extensor torques at the shoulder and flexor torques at the elbow $\left(\mathrm{PTD}=321^{\circ}\right.$, Figure 4C). Similarly, an exemplar EMG recording from brachioradialis showed increases in activity for responses requiring flexor torques at the elbow and extensor torques at the shoulder $\left(\mathrm{PTD}=105^{\circ}\right.$, Figure 4D). Both of these patterns are consistent with activities that would bring the hand back to the target in response to their stretch. Both muscles exhibited narrow tuning relative to a cosine (tuning widths $=0.16$ and 0.25 , Figures 4C,D, right). Both muscles also showed far greater excitation than inhibition, relative to their baseline activities (excitation-inhibition ratios $=1.06$ and 0.62 , Figures 4C,D, left).

\section{Comparison of Onset Latencies}

We compared the response latencies of $\mathrm{RN}$ neurons, M1 neurons and upper limb muscles (Figure 5). As indicated above, many RN neurons (57\%), M1 neurons (39\%) and upperlimb muscles (67\%) exhibited rapid responses (20-100 ms), suggesting that their activity is tightly coupled to the mechanical (sensory) stimulus. We found that the mean onset latencies of $\mathrm{RN}(44 \pm 14 \mathrm{~ms})$ and $\mathrm{M} 1(46 \pm 19 \mathrm{~ms})$ neurons were not significantly different from each other (Wilcoxon rank sum test, $p>0.1$ ). By comparison, the mean onset latency of upper-limb muscles (55 $\pm 22 \mathrm{~ms}$ ) was significantly longer than RN neurons (one-tailed $t$-test, $p<0.05$ ) but did not differ significantly from M1 neurons, (Wilcoxon rank sum test, $p>0.1)$.

\section{Comparison of Tuning Properties}

A common characteristic of M1 neurons is that their torquerelated activity exhibits a bimodal distribution of PTDs that mirrors the distribution observed in upper-limb muscles (reviewed in Kurtzer and Scott, 2007). Specifically, PTDs of M1 neurons and upper-limb muscles are both biased towards whole-limb flexor $(\mathrm{EF}+\mathrm{SE})$ and whole-limb extensor $(\mathrm{EE}+\mathrm{SF})$ torques. Figure 6 investigates whether the RN neurons, M1 neurons and upper-limb muscles examined in the current study exhibit similar bimodal distributions of PTDs. Consistent with our previous studies, bimodal distributions of PTDs were seen in M1 neurons (Figure 6B, unimodal Rayleigh test, $p>0.1$; bimodal Rayleigh test, $p<0.01, r=0.53$, PTD axes $\left.=132-312^{\circ}\right)$ and upper-limb muscles (Figure 6C, unimodal Rayleigh test, $p>0.1$; bimodal Rayleigh test, $p<0.01, r=0.66$, PTD axes $=125-305^{\circ}$ ). In contrast, $\mathrm{RN}$ neurons exhibited similar results for unimodality and bimodality. RN neurons exhibited a unimodal distribution that was marginally insignificant (unimodal Rayleigh test, 

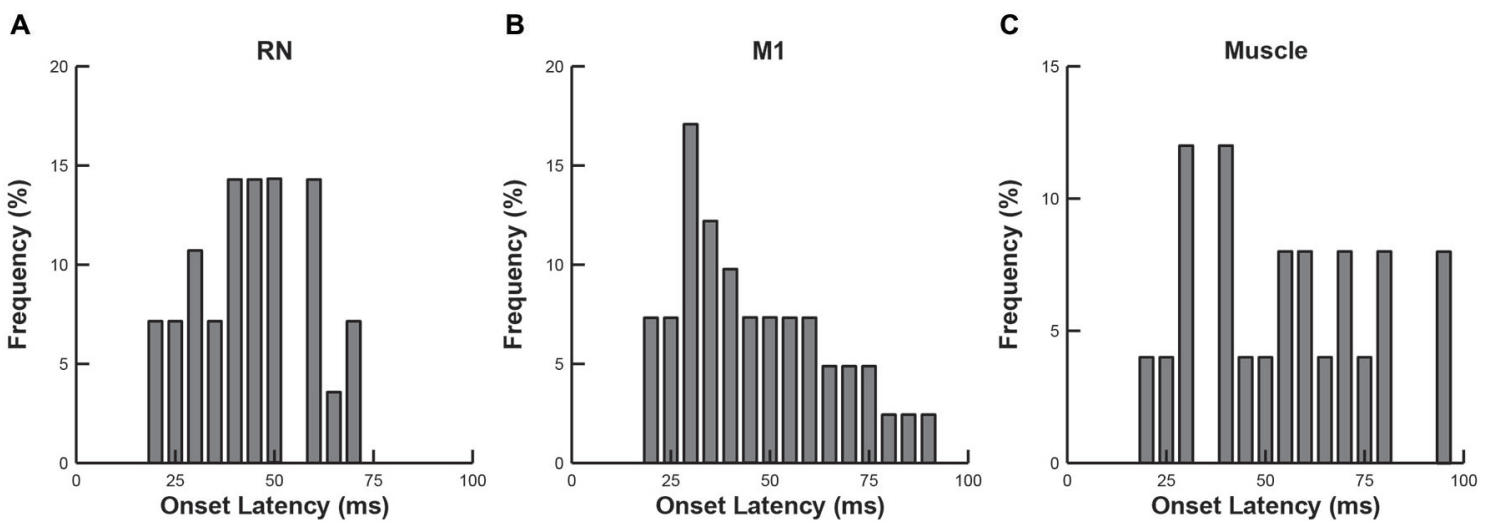

FIGURE 5 | Frequency histograms of onset latencies. (A) RN neurons. (B) M1 neurons. (C) Upper-limb muscles.
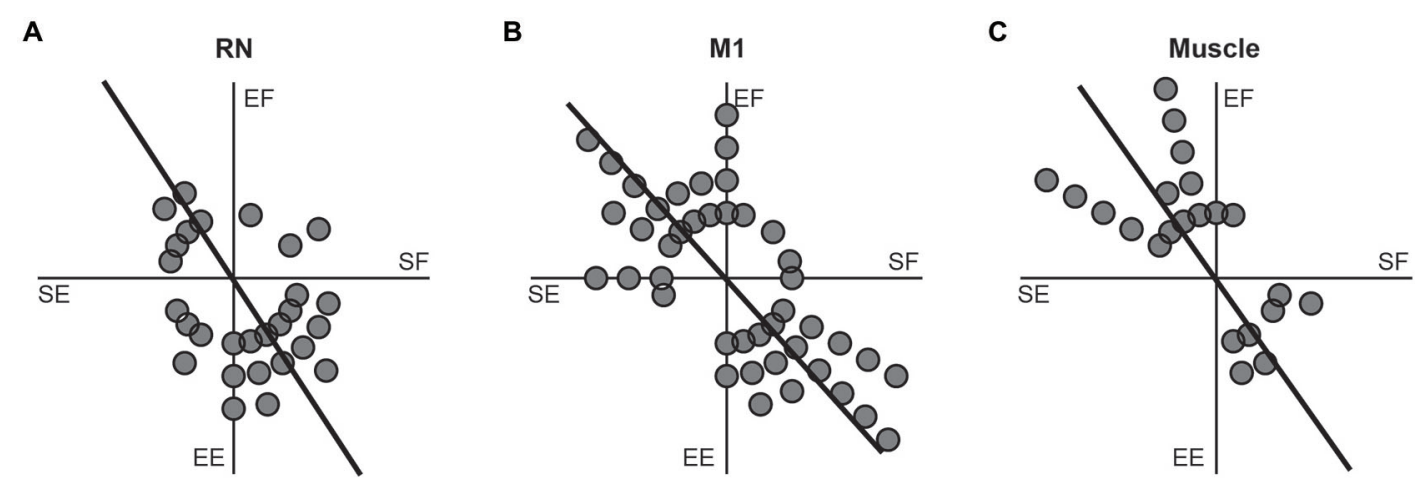

FIGURE 6 | Frequency histograms of preferred-torque directions. (A) RN neurons. (B) M1 neurons. (C) Upper-limb muscles. Each filled circle shows one neuron or muscle with a PTD within a $15^{\circ}$ bin. Thick lines show the bimodal axes for each distribution of PTDs.

$\left.p=0.06, r=0.31, \mathrm{PTD}=288^{\circ}\right)$, whereas the bimodal distribution was marginally significant (Figure 6A; bimodal Rayleigh test, $p<0.05, r=0.33$, PTD axes $\left.=123-303^{\circ}\right)$. Importantly, the distributions of RN neurons, M1 neurons and upper-limb muscles did not differ significantly from one another ( $t$-tests, all $p>0.1$ ).

In addition to comparing PTDs, we also examined whether the temporal evolution of other directional tuning properties in $\mathrm{RN}$ and $\mathrm{M} 1$ were similar to upper-limb muscles. Note that, because of the difference in units, TSs of neurons (discharge frequency) could not be directly compared with TSs obtained from EMG activity in muscles (arbitrary units). However, we found the mean TS in both brain regions and upper-limb muscles increased over time following perturbations (Figure 7A, linear regressions, all $p<0.05)$. Furthermore, the mean TS in $\mathrm{RN}$ was significantly greater than in M1 (Figure 7A, ANOVA, $p<0.01$ ), indicating that firing frequencies of $\mathrm{RN}$ neurons increased more than M1 neurons in response to perturbations. Comparisons of tuning widths revealed that tuning widths were significantly narrower in upper-limb muscles than neurons in M1 and RN (ANOVA, $p<0.01$ ), though the tuning widths were generally narrower than cosine tuning in all three (Figure 7B). Finally, we found that EIRs were broadly distributed in both RN and M1 (Figure 7C), including many neurons that exhibited reciprocal excitation and inhibition $(-1<$ EIR $<1)$ and many neurons that were excited by all perturbations but in differing amounts $($ EIR $>1)$. By comparison, most muscles exhibited reciprocal excitation and inhibition in which excitation was greater than inhibition $(0<\mathrm{EIR}<1)$. Despite these differences in the breadth of excitation and inhibition, we did not observe a significant difference in EIRs between RN neurons, M1 neurons and upperlimb muscles (Figure 7C, ANOVA, $p>0.1$ ).

\section{Discussion}

The goal of the present study was to investigate whether mechanical perturbations evoke rapid sensorimotor responses in $\mathrm{RN}$ neurons that are similar to those observed in M1 neurons and upper-limb muscles. In general, perturbation responses in $\mathrm{RN}$ neurons were qualitatively similar to those observed in M1, with broad tuning and preferred torque directions biased towards whole limb flexor torques (elbow flexor and shoulder extensor) and whole limb extensor torques (elbow extensor and shoulder flexor). Timing of perturbation responses was also 
A

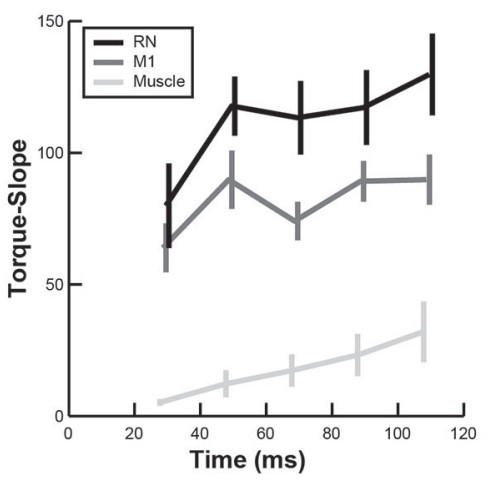

B

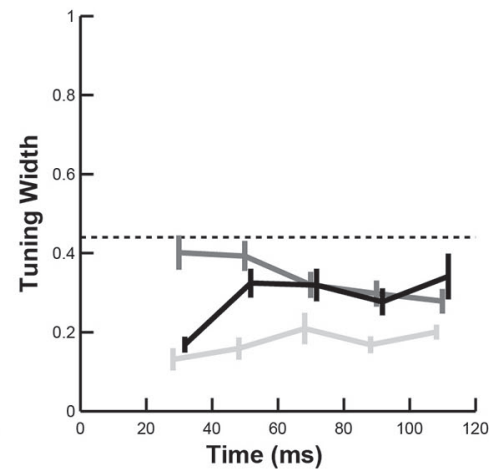

C

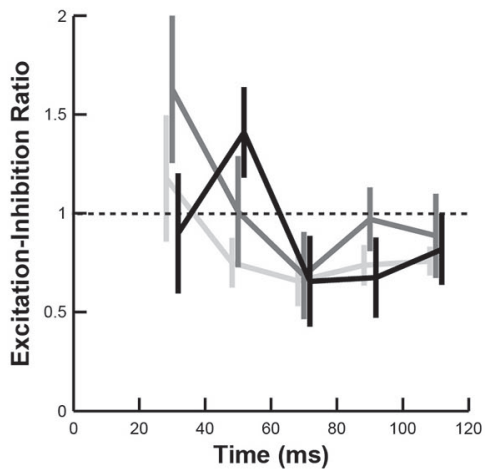

FIGURE 7 | Temporal evolution of perturbation-related activity. (A-C) Plots show changes in torque-slope (A), tuning width (B) and activation-inhibition ratio (C) of RN neurons (black), M1 neurons (dark gray) and upper-limb muscles (light gray) during the first 120 ms after perturbation onset (mean \pm sem).

similar across the two regions, with onsets beginning at $\sim 20 \mathrm{~ms}$ after perturbations were applied. However, RN neurons tended to display larger perturbation responses than M1 neurons with regards to the absolute change in discharge.

Both RN and M1 exhibited distributions of PTDs that were skewed towards one of two quadrants in joint-torque space, shoulder extensor torque coupled with elbow flexor torque (whole-limb flexor torque) and shoulder flexor torque coupled with elbow extensor torque (whole-limb extensor torque) (Figures 6A,B). However, RN neurons exhibited similar vector lengths for the unimodal $(r=0.31)$ and bimodal distributions $(r=0.32)$. The unimodal PTD of $288^{\circ}$ indicates that more RN neurons were related to whole-limb extensor torques, which is consistent with several studies on RN (Gibson et al., 1985a; Cheney et al., 1988; Mewes and Cheney, 1994; Sinkjaer et al., 1995; Belhaj-Saif et al., 1998; Park et al., 2004). Similar to M1 and RN, PTDs of shoulder and elbow muscles were also skewed towards whole-limb flexor and whole-limb extensor torques (Figure 6C) even though some muscles spanned only one joint (i.e., monoarticular) and those muscles that spanned both joints (i.e., biarticular muscles) possess pulling actions in the opposite quadrants (Graham and Scott, 2003). A similar bias has also been observed in M1 and upper-limb muscles when continuous loads were applied to the shoulder and/or elbow during static posture (Cabel et al., 2001; Kurtzer et al., 2005, 2006a; Herter et al., 2007, 2009), as well as for dynamic or static loads applied to the limb during reaching (Gribble and Scott, 2002; Kurtzer et al., 2005, 2006b).

These biases in the distribution of PTDs exhibited by $\mathrm{RN}$ and M1 neurons appear to reflect the anatomical properties of the musculoskeletal system (Kurtzer et al., 2006a,b; Lillicrap and Scott, 2013). Both mathematical and neural network models highlight that the bias in the distribution of PTDs was only observed when biarticular muscles were included in the models. This provides strong evidence that the patterns of activity observed in RN and M1 neurons reflect constraints imposed by the anatomical organization of the musculoskeletal system (Kurtzer and Scott, 2007).
In theory, proximal limb muscles should exhibit relatively rapid perturbation responses (within $25 \mathrm{~ms}$ ) due to spinal level feedback. However, only a few muscles displayed perturbation responses below $30 \mathrm{~ms}$. The late muscle responses likely reflect the fact that minimal muscle activity is required to overcome passive limb forces in the middle of the workspace (Graham et al., 2003). Short latency stretch responses increase with baseline activity, but are small or not present when the muscle is inactive prior to the perturbation (Pruszynski et al., 2011a). Muscles were also commonly modulated by only two to three load conditions, whereas neurons in $\mathrm{RN}$ and M1 were generally modulated by three to four load conditions. Stated otherwise, muscles were rarely activated by flexor and extensor torques at a joint, whereas neurons were commonly activated by flexor and extensor torques at one of the two joints.

Perhaps the largest difference between RN and M1 neurons was in their mean discharge rates, as measured by TSs (Figure 7A). Although neurons in both structures exhibited steady increases in TS over the first $100 \mathrm{~ms}$ following perturbations (mirroring upper-limb muscles), the neurons in RN exhibited systematically higher TS values than neurons in M1. Perturbation responses were approximately 50\% larger in $\mathrm{RN}$ neurons as compared to M1 neurons. Higher firing rates have also been observed in brainstem regions (superior colliculus) compared to cortical regions (frontal eye fields) during saccadic eye movements (Jantz et al., 2013).

The present study shows that both $\mathrm{M} 1$ and $\mathrm{RN}$ receive rapid feedback from the motor periphery. The dorsal column system provides the primary source of feedback to M1, including direct inputs from thalamus and indirect inputs via primary somatosensory cortex (Brinkman et al., 1978; Horne and Tracey, 1979; Asanuma et al., 1980). Projections from cerebellum also contribute sensory information to M1 (Massion, 1976; Asanuma et al., 1983; Butler et al., 1992). The dorsal column system is also the principle source of sensory information for $\mathrm{RN}$ (Berkley et al., 1986; Boivie, 1988). Importantly, $\mathrm{RN}$ is a site of significant convergence on sensory and motor inputs from both cortex and cerebellum, suggesting that M1, RN 
and cerebellum form a recurrent network that is involved in feedback control of voluntary motor actions. This is further supported by observations that perturbation-related activity in M1 is modulated by behavioral context (Conrad et al., 1974, 1975; Evarts and Tanji, 1974, 1976; Wolpaw, 1980b; Omrani et al., 2014; Pruszynski et al., 2014). It remains to be explored if perturbation responses in RN are similarly modulated by the behavioral goal.

The RN contains two regions, magnocellular and parvocellular, with the latter projecting principally to the inferior olivary nucleus creating a circuit with the cerebellum, and the former providing the origin of the rubrospinal tract (Houk et al., 1993). The corticospinal tract is much larger than rubrospinal tract in non-human primates and this difference is even greater in humans (Larsen and Yumiya, 1980; Nathan and Smith, 1982). Although we did not identify whether our sample of $\mathrm{RN}$ neurons were in the magnocellular or parvocellular regions of $\mathrm{RN}$, it would be interesting to know if there was any

\section{References}

Asanuma, H., Larsen, K., and Yumiya, H. (1980). Peripheral input pathways to the monkey motor cortex. Exp. Brain Res. 38, 349-355. doi: 10.1007/bf00236655

Asanuma, C., Thach, W. T., and Jones, E. G. (1983). Distribution of cerebellar terminations and their relation to other afferent terminations in the ventral lateral thalamic region of the monkey. Brain Res. 286, 237-265. doi: 10. 1016/0165-0173(83)90015-2

Batschelet, E. (1981). Circular Statistics in Biology. New York: Academic Press.

Bauswein, E., Fromm, C., Werner, W., and Ziemann, U. (1991). Phasic and tonic responses of premotor and primary motor cortex neurons to torque changes. Exp. Brain Res. 86, 303-310. doi: 10.1007/bf00228953

Belhaj-Saif, A., Karrer, J. H., and Cheney, P. D. (1998). Distribution and characteristics of poststimulus effects in proximal and distal forelimb muscles from red nucleus in the monkey. J. Neurophysiol. 79, 1777-1789.

Bennett, K. M., and Lemon, R. N. (1996). Corticomotoneuronal contribution to the fractionation of muscle activity during precision grip in the monkey. J. Neurophysiol. 75, 1826-1842.

Berkley, K. J., Budell, R. J., Blomqvist, A., and Bull, M. (1986). Output systems of the dorsal column nuclei in the cat. Brain Res. 396, 199-225. doi: 10.1016/01650173(86)90012-3

Boivie, J. (1988). Projections from the dorsal column nuclei and the spinal cord to the red nucleus in cat. Behav. Brain Res. 28, 75-79. doi: 10.1016/01664328(88)90080-0

Brinkman, J., Bush, B. M., and Porter, R. (1978). Deficient influence of peripheral stimuli on precentral neurones in monkeys with dorsal column lesions. J. Physiol. 276, 27-48. doi: 10.1113/jphysiol.1978.sp012218

Butler, E. G., Horne, M. K., and Rawson, J. A. (1992). Sensory characteristics of monkey thalamic and motor cortex neurones. J. Physiol. 445, 1-424. doi: 10. 1113/jphysiol.1992.sp018909

Buys, E. J., Lemon, R. N., Mantel, G. W., and Muir, R. B. (1986). Selective facilitation of different hand muscles by single corticospinal neurones in the conscious monkey. J. Physiol. 381, 529-549. doi: 10.1113/jphysiol.1986. sp016342

Cabel, D. W., Cisek, P., and Scott, S. H. (2001). Neural activity in primary motor cortex related to mechanical loads applied to the shoulder and elbow during a postural task. J. Neurophysiol. 86, 2102-2108.

Cheney, P. D., and Fetz, E. E. (1984). Corticomotoneuronal cells contribute to long-latency stretch reflexes in the rhesus monkey. J. Physiol. 349, 249-272. doi: 10.1113/jphysiol.1984.sp015155

Cheney, P. D., Fetz, E. E., and Mewes, K. (1991). Neural mechanisms underlying corticospinal and rubrospinal control of limb movements. Prog. Brain Res. 87, 213-252. doi: 10.1016/s0079-6123(08)63054-x substantive difference in the perturbation response properties in these sub-regions of RN.

\section{Author Contributions}

TMH helped design the study, performed the data analysis, and wrote the manuscript. TT assisted with the data analysis. DPM helped design the study and assisted with data collection. SHS helped design the study, assisted with the data analysis, and participated in writing of the manuscript. All authors approved the final version of the manuscript.

\section{Acknowledgments}

This research was supported by a Canadian Institutes of Health Research (CIHR) grant and a CIHR Investigator award to SHS. We wish to thank Kim Moore, Jennifer Green, Helen Bretzke, and Justin Peterson for their technical support.

Cheney, P. D., Mewes, K., and Fetz, E. E. (1988). Encoding of motor parameters by corticomotoneuronal (CM) and rubromotoneuronal (RM) cells producing postspike facilitation of forelimb muscles in the behaving monkey. Behav. Brain Res. 28, 181-191. doi: 10.1016/0166-4328(88)90095-2

Cluff, T., and Scott, S. H. (2013). Rapid feedback responses correlate with reach adaptation and properties of novel upper limb loads. J. Neurosci. 33, 15903-15914. doi: 10.1523/jneurosci.0263-13.2013

Conrad, B., Matsunami, K., Meyer-Lohmann, J., Wiesendanger, M., and Brooks, V. B. (1974). Cortical load compensation during voluntary elbow movement. Brain Res. 71, 507-514. doi: 10.1016/0006-8993(74)90994-9

Conrad, B., Meyer-Lohmann, J., Matsunami, K., and Brooks, V. B. (1975). Precentral unit activity following torque pulse injections into elbow movements. Brain Res. 94, 219-236. doi: 10.1016/0006-8993(75) 90058-x

Crevecoeur, F., Kurtzer, I., Bourke, T., and Scott, S. H. (2013). Feedback responses rapidly scale with the urgency to correct for external perturbations. J. Neurophysiol. 110, 1323-1332. doi: 10.1152/jn.00216.2013

Dimitriou, M., Franklin, D. W., and Wolpert, D. M. (2012). Task-dependent coordination of rapid bimanual motor responses. J. Neurophysiol. 107, 890-901. doi: 10.1152/jn.00787.2011

Evarts, E. V. (1973). Motor cortex reflexes associated with learned movement. Science. 179, 501-503. doi: 10.1126/science.179.4072.501

Evarts, E. V., and Fromm, C. (1977). Sensory responses in motor cortex neurons during precise motor control. Neurosci. Lett. 5, 267-272. doi: 10.1016/03043940(77)90077-5

Evarts, E. V., and Tanji, J. (1974). Gating of motor cortex reflexes by prior instruction. Brain Res. 71, 479-494. doi: 10.1016/0006-8993(74)90992-5

Evarts, E. V., and Tanji, J. (1976). Reflex and intended responses in motor cortex pyramidal tract neurons of monkey. J. Neurophysiol. 39, 1069-1080.

Fetz, E. E., and Cheney, P. D. (1980). Postspike facilitation of forelimb muscle activity by primate corticomotoneuronal cells. J. Neurophysiol. 44, 751-772.

Fetz, E. E., Finocchio, D. V., Baker, M. A., and Soso, M. J. (1980). Sensory and motor responses of precentral cortex cells during comparable passive and active joint movements. J. Neurophysiol. 43, 1070-1089.

Flament, D., and Hore, J. (1988). Relations of motor cortex neural discharge to kinematics of passive and active elbow movements in the monkey. J. Neurophysiol. 60, 1268-1284.

Fromm, C., Wise, S. P., and Evarts, E. V. (1984). Sensory response properties of pyramidal tract neurons in the precentral motor cortex and postcentral gyrus of the rhesus monkey. Exp. Brain Res. 54, 177-185. doi: 10.1007/bf00 235829

Gibson, A. R., Houk, J. C., and Kohlerman, N. J. (1985a). Magnocellular red nucleus activity during different types of limb movement in the macaque monkey. J. Physiol. 358, 527-549. doi: 10.1113/jphysiol.1985.sp015565 
Gibson, A. R., Houk, J. C., and Kohlerman, N. J. (1985b). Relation between red nucleus discharge and movement parameters in trained macaque monkeys. J. Physiol. 358, 551-570. doi: 10.1113/jphysiol.1985.sp015566

Graham, K. M., Moore, K. D., Cabel, D. W., Gribble, P. L., Cisek, P., and Scott, S. H. (2003). Kinematics and kinetics of multijoint reaching in nonhuman primates. J. Neurophysiol. 89, 2667-2677. doi: 10.1152/jn.00742.2002

Graham, K. M., and Scott, S. H. (2003). Morphometry of Macaca mulatta forelimb. III. Moment arm of shoulder and elbow muscles. J. Morphol. 255, 301-314. doi: 10.1002/jmor.10064

Gribble, P. L., and Scott, S. H. (2002). Overlap of internal models in motor cortex for mechanical loads during reaching. Nature 417, 938-941. doi: 10. 1038/nature00834

Herter, T. M., Korbel, T., and Scott, S. H. (2009). Comparison of neural responses in primary motor cortex to transient and continuous loads during posture. J. Neurophysiol. 101, 150-163. doi: 10.1152/jn.90230.2008

Herter, T. M., Kurtzer, I., Cabel, D. W., Haunts, K. A., and Scott, S. H. (2007). Characterization of torque-related activity in primary motor cortex during a multijoint postural task. J. Neurophysiol. 97, 2887-2899. doi: 10.1152/jn.00757. 2006

Holdefer, R. N., and Miller, L. E. (2002). Primary motor cortical neurons encode functional muscle synergies. Exp. Brain Res. 146, 233-243. doi: 10.1007/s00221002-1166-x

Horne, M. K., and Tracey, D. J. (1979). The afferents and projections of the ventroposterolateral thalamus in the monkey. Exp. Brain Res. 36, 129-141. doi: $10.1007 /$ bf00238473

Houk, J. C., Keifer, J., and Barto, A. G. (1993). Distributed motor commands in the limb premotor network. Trends Neurosci. 16, 27-33. doi: 10.1016/01662236(93)90049-r

Jantz, J. J., Watanabe, M., Everling, S., and Munoz, D. P. (2013). Threshold mechanism for saccade initiation in frontal eye field and superior colliculus. J. Neurophysiol. 109, 2767-2780. doi: 10.1152/jn.00611.2012

Keifer, J., and Houk, J. C. (1994). Motor function of the cerebellorubrospinal system. Physiol. Rev. 74, 509-542.

Kennedy, P. R. (1987). Parametric relationships of individual digit movements to neuronal discharges in primate magnocellular red nucleus. Brain Res. 417, 185-189. doi: 10.1016/0006-8993(87)90198-3

Kennedy, P. R. (1990). Corticospinal, rubrospinal and rubro-olivary projections: a unifying hypothesis. Trends Neurosci. 13, 474-479. doi: 10.1016/01662236(90)90079-p

Kennedy, P. R., Gibson, A. R., and Houk, J. C. (1986). Functional and anatomic differentiation between parvicellular and magnocellular regions of red nucleus in the monkey. Brain Res. 364, 124-136. doi: 10.1016/0006-8993 (86)90993-5

Kohlerman, N. J., Gibson, A. R., and Houk, J. C. (1982). Velocity signals related to hand movements recorded from red nucleus neurons in monkeys. Science 217, 857-860. doi: 10.1126/science.7100930

Kurtzer, I., Crevecoeur, F., and Scott, S. H. (2014). Fast feedback control involves two independent processes utilizing knowledge of limb dynamics. J. Neurophysiol. 111, 1631-1645. doi: 10.1152/jn.00514.2013

Kurtzer, I., Herter, T. M., and Scott, S. H. (2005). Random change in cortical load representation suggests distinct control of posture and movement. Nat. Neurosci. 8, 498-504. doi: 10.1038/nn1420

Kurtzer, I., Herter, T. M., and Scott, S. H. (2006b). Nonuniform distribution of reach-related and torque-related activity in upper arm muscles and neurons of primary motor cortex. J. Neurophysiol. 96, 3220-3230. doi: 10.1152/jn.00110. 2006

Kurtzer, I., Pruszynski, J. A., Herter, T. M., and Scott, S. H. (2006a). Primate upper limb muscles exhibit activity patterns that differ from their anatomical action during a postural task. J. Neurophysiol. 95, 493-504. doi: 10.1152/jn.00706.2005

Kurtzer, I., Pruszynski, J. A., and Scott, S. H. (2009). Long-latency responses during reaching account for the mechanical interaction between the shoulder and elbow joints. J. Neurophysiol. 102, 3004-3015. doi: 10.1152/jn.00453.2009

Kurtzer, I. L., Pruszynski, J. A., and Scott, S. H. (2008). Long-latency reflexes of the human arm reflect an internal model of limb dynamics. Curr. Biol. 18, 449-453. doi: 10.1016/j.cub.2008.02.053

Kurtzer, I., and Scott, S. H. (2007). A multi-level approach to understanding upper limb function. Prog. Brain Res. 165, 347-362. doi: 10.1016/s00796123(06)65021-8
Larsen, K. D., and Yumiya, H. (1980). The red nucleus of the monkey. Topographic localization of somatosensory input and motor output. Exp. Brain Res. 40, 393-404.

Lemon, R. N. (1981). Functional properties of monkey motor cortex neurones receiving afferent input from the hand and fingers. J. Physiol. 311, 497-519. doi: 10.1113/jphysiol.1981.sp013601

Lillicrap, T. P., and Scott, S. H. (2013). Preference distributions of primary motor cortex neurons reflect control solutions optimized for limb biomechanics Neuron 77, 168-179. doi: 10.1016/j.neuron.2012.10.041

Loeb, G. E., and Gans, C. (1986). Electromyography for Experimentalists. London: University of Chicago Press.

Marino, R. A., Rodgers, C. K., Levy, R., and Munoz, D. P. (2008). Spatial relationships of visuomotor transformations in the superior colliculus map. J. Neurophysiol. 100, 2564-2576. doi: 10.1152/jn.90688.2008

Massion, J. (1976). The thalamus in the motor system. Appl. Neurophysiol. 39, 222-238. doi: 10.1159/000102498

McKiernan, B. J., Marcario, J. K., Karrer, J. H., and Cheney, P. D. (1998). Corticomotoneuronal postspike effects in shoulder, elbow, wrist, digit and intrinsic hand muscles during a reach and prehension task. J. Neurophysiol. 80, 1961-1980.

Mewes, K., and Cheney, P. D. (1991). Facilitation and suppression of wrist and digit muscles from single rubromotoneuronal cells in the awake monkey. J. Neurophysiol. 66, 1965-1977.

Mewes, K., and Cheney, P. D. (1994). Primate rubromotoneuronal cells: parametric relations and contribution to wrist movement. J. Neurophysiol. 72, $14-30$.

Miller, L. E., and Houk, J. C. (1995). Motor co-ordinates in primate red nucleus: preferential relation to muscle activation versus kinematic variables. J. Physiol. 488, 533-548. doi: 10.1113/jphysiol.1995.sp020988

Miller, L. E., and Sinkjaer, T. (1998). Primate red nucleus discharge encodes the dynamics of limb muscle activity. J. Neurophysiol. 80, 59-70.

Miller, L. E., van Kan, P. L., Sinkjaer, T., Andersen, T., Harris, G. D., and Houk, J. C. (1993). Correlation of primate red nucleus discharge with muscle activity during free-form arm movements. J. Physiol. 469, 213-243. doi: 10. 1113/jphysiol.1993.sp019812

Nashed, J. Y., Crevecoeur, F., and Scott, S. H. (2012). Influence of the behavioral goal and environmental obstacles on rapid feedback responses. J. Neurophysiol. 108, 999-1009. doi: 10.1152/jn.01089.2011

Nashed, J. Y., Crevecoeur, F., and Scott, S. H. (2014). Rapid online selection between multiple motor plans. J. Neurosci. 34, 1769-1780. doi: 10 . 1523/JNEUROSCI.3063-13.2014

Nathan, P. W., and Smith, M. C. (1982). The rubrospinal and central tegmental tracts in man. Brain 105, 223-269. doi: 10.1093/brain/105.2.223

Omrani, M., Diedrichsen, J., and Scott, S. H. (2013). Rapid feedback corrections during a bimanual postural task. J. Neurophysiol. 109, 147-161. doi: 10.1152/jn. 00669.2011

Omrani, M., Pruszynski, J. A., Murnaghan, C. D., and Scott, S. H. (2014). Perturbation-evoked responses in primary motor cortex are modulated by behavioral context. J. Neurophysiol. 112, 2985-3000. doi: 10.1152/jn.00270. 2014

Park, M. C., Belhaj-Saï, A., and Cheney, P. D. (2004). Properties of primary motor cortex output to forelimb muscles in rhesus macaques. J. Neurophysiol. 92, 2968-2984. doi: 10.1152/jn.00649.2003

Pruszynski, J. A., Kurtzer, I., Nashed, J. Y., Omrani, M., Brouwer, B., and Scott, S. H. (2011b). Primary motor cortex underlies multi-joint integration for fast feedback control. Nature 478, 387-390. doi: 10.1038/nature10436

Pruszynski, J. A., Kurtzer, I., and Scott, S. H. (2008). Rapid motor responses are appropriately tuned to the metrics of a visuo-spatial task. J. Neurophysiol. 100, 224-238. doi: 10.1152/jn.90262.2008

Pruszynski, J. A., Kurtzer, I., and Scott, S. H. (2011a). The long-latency reflex is composed of at least two functionally independent processes. J. Neurophysiol. 106, 449-459. doi: 10.1152/jn.01052.2010

Pruszynski, J. A., Omrani, M., and Scott, S. H. (2014). Goal-dependent modulation of fast feedback responses in primary motor cortex. J. Neurosci. 34, 4608-4617. doi: 10.1523/JNEUROSCI.4520-13.2014

Scott, S. H. (1997). Comparison of onset time and magnitude of activity for proximal arm muscles and motor cortical cells prior to reaching movements. J. Neurophysiol. 77, 1016-1022. 
Scott, S. H. (1999). Apparatus for measuring and perturbing shoulder and elbow joint positions and torques during reaching. J. Neurosci. Methods 89, 119-127. doi: 10.1016/s0165-0270(99)00053-9

Scott, S. H. (2003). The role of primary motor cortex in goal-directed movements: insights from neurophysiological studies on non-human primates. Curr. Opin. Neurobiol. 13, 671-677. doi: 10.1016/j.conb.2003.10.012

Scott, S. H. (2004). Optimal feedback control and the neural basis of volitional motor control. Nat. Rev. Neurosci. 5, 532-546. doi: 10.1038/nrn1427

Scott, S. H. (2012). The computational and neural basis of voluntary motor control and planning. Trends Cogn. Sci. 16, 541-549. doi: 10.1016/j.tics.2012. 09.008

Scott, S. H., and Kalaska, J. F. (1997). Reaching movements with similar hand paths but different arm orientations: I. Activity of individual cells in motor cortex. J. Neurophysiol. 77, 826-852.

Sinkjaer, T., Miller, L., Andersen, T., and Houk, J. C. (1995). Synaptic linkages between red nucleus cells and limb muscles during a multi-joint motor task. Exp. Brain Res. 102, 546-550. doi: 10.1007/bf00230659

Smith, A. M., Hepp-Reymond, M. C., and Wyss, U. R. (1975). Relation of activity in of precentral cortical neurons to force and rate of force change during isometric contractions of finger muscles. Exp. Brain Res. 23, 315-332. doi: 10. 1007/bf00239743

Thompson, K. G., Hanes, D. P., Bichot, N. P., and Schall, J. D. (1996). Perceptual and motor processing stages identified in the activity of macaque frontal eye field neurons during visual search. J. Neurophysiol. 76, 4040-4055.

Todorov, E. (2004). Optimality principles in sensorimotor control. Nat. Neurosci. 7, 907-915. doi: 10.1038/nn1309
Todorov, E., and Jordan, M. I. (2002). Optimal feedback control as a theory of motor coordination. Nat. Neurosci. 5, 1226-1235. doi: 10.1038/nn963

van Kan, P. L., and McCurdy, M. L. (2001). Role of primate magnocellular red nucleus neurons in controlling hand preshaping during reaching to grasp. J. Neurophysiol. 85, 1461-1478.

van Kan, P. L., and McCurdy, M. L. (2002a). Discharge of primate magnocellular red nucleus neurons during reaching to grasp in different spatial locations. Exp. Brain Res. 142, 151-157. doi: 10.1007/s00221-001-0924-5

van Kan, P. L., and McCurdy, M. L. (2002b). Role of primate magnocellular red nucleus neurons in controlling hand preshaping during reaching to grasp. J. Neurophysiol. 85, 1461-1478.

Wolpaw, J. R. (1980a). Correlations between task-related activity and responses to perturbation in primate sensorimotor cortex. J. Neurophysiol. 44, 1122-1138.

Wolpaw, J. R. (1980b). Amplitude of responses to perturbation in primate sensorimotor cortex as a function of task. J. Neurophysiol. 44, 1139-1147.

Conflict of Interest Statement: SHS is the Co-founder and Chief Scientific Officer of BKIN Technologies that commercializes the robotic technology used in this study.

Copyright (C) 2015 Herter, Takei, Munoz and Scott. This is an open-access article distributed under the terms of the Creative Commons Attribution License (CC BY). The use, distribution and reproduction in other forums is permitted, provided the original author(s) or licensor are credited and that the original publication in this journal is cited, in accordance with accepted academic practice. No use, distribution or reproduction is permitted which does not comply with these terms. 